\title{
Higgs bosons decaying into 4 fermions in the Two-Higgs-Doublet Model
}

\section{Lukas Altenkamp}

Albert-Ludwigs-Universität Freiburg, Physikalisches Institut, Freiburg, Germany

\section{Stefan Dittmaier}

Albert-Ludwigs-Universität Freiburg, Physikalisches Institut, Freiburg, Germany

E-mail: stefan.dittmaier@physik.uni-freiburg.de

\section{Heidi Rzehak*}

$C P^{3}$-Origins, University of Southern Denmark, Odense, Denmark

E-mail: rzehak@cp3.sdu.dk

\begin{abstract}
We present the next-to-leading-order calculation of the partial decay widths of a Higgs boson decaying into four fermions in the Two-Higgs-Doublet Model, including a discussion of different renormalization schemes. We also comment on new developments in the renormalization of mixing angles. The calculation is implemented into the analysis tool PROPHECY4F, and some sample results generated with the extension of PROPHECY4F are shown.
\end{abstract}

Corfu Summer Institute 2018 "School and Workshops on Elementary Particle Physics and Gravity" (CORFU2018)

31 August - 28 September, 2018

Corfu, Greece

${ }^{*}$ Speaker. 


\section{Introduction}

The Higgs-boson decay to four fermions is one of the best studied Higgs decay channels. Among these channels, the decay into four charged leptons is a very clean signal and plays a crucial role in the Higgs mass measurement [1]. In order to exploit an improved accuracy of the measurements, not only theoretical predictions of the Standard Model (SM) but also of possible extensions of the SM need to be improved to the same level of precision. Therefore, higher-order corrections need to be incorporated.

One of the simplest extensions of the SM is the Two-Higgs-Doublet Model (THDM) where only a Higgs doublet is added to the SM, giving rise to additional Higgs bosons, two neutral and one charged Higgs boson. For many more complex models that contain a second Higgs doublet a general THDM is a low-energy effective theory.

We present the calculation of the Higgs-boson decay to four fermions in the THDM at nextto-leading order (NLO) including electroweak (EW) as well as QCD corrections, restricting the calculation to a THDM with the specific assumptions described below.

\section{The Two-Higgs-Doublet Model and its renormalization}

We consider the THDM Higgs potential

$$
\begin{aligned}
V= & m_{11}^{2} \Phi_{1}^{\dagger} \Phi_{1}+m_{22}^{2} \Phi_{2}^{\dagger} \Phi_{2}-m_{12}^{2}\left(\Phi_{1}^{\dagger} \Phi_{2}+\Phi_{2}^{\dagger} \Phi_{1}\right) \\
& +\frac{1}{2} \lambda_{1}\left(\Phi_{1}^{\dagger} \Phi_{1}\right)^{2}+\frac{1}{2} \lambda_{2}\left(\Phi_{2}^{\dagger} \Phi_{2}\right)^{2}+\lambda_{3}\left(\Phi_{1}^{\dagger} \Phi_{1}\right)\left(\Phi_{2}^{\dagger} \Phi_{2}\right)+\lambda_{4}\left(\Phi_{1}^{\dagger} \Phi_{2}\right)\left(\Phi_{2}^{\dagger} \Phi_{1}\right) \\
& +\frac{1}{2} \lambda_{5}\left[\left(\Phi_{1}^{\dagger} \Phi_{2}\right)^{2}+\left(\Phi_{2}^{\dagger} \Phi_{1}\right)^{2}\right]
\end{aligned}
$$

where $\Phi_{1}, \Phi_{2}$ denote the two Higgs doublets, $m_{11}^{2}, m_{12}^{2}, m_{22}^{2}$ the mass parameters, and $\lambda_{1}, \ldots, \lambda_{5}$ the quartic Higgs couplings. The symmetry of the Higgs potential under the transformation $\Phi_{1} \rightarrow$ $-\Phi_{1}$ is only softly broken by non-vanishing values of $m_{12}^{2}$ [2, 3]. In addition, CP-conservation is assumed so that all parameters in the Higgs potential are real. The two Higgs doublets can be decomposed as

$$
\Phi_{1}=\left(\begin{array}{c}
\phi_{1}^{+} \\
\frac{1}{\sqrt{2}}\left(\eta_{1}+i \chi_{1}+v_{1}\right)
\end{array}\right), \quad \Phi_{2}=\left(\begin{array}{c}
\phi_{2}^{+} \\
\frac{1}{\sqrt{2}}\left(\eta_{2}+i \chi_{2}+v_{2}\right)
\end{array}\right),
$$

where $v_{1}$ and $v_{2}$ are the Higgs vacuum expectation values and $\phi_{1}^{+}, \phi_{2}^{+}, \eta_{1}, \eta_{2}, \chi_{1}, \chi_{2}$ the charged, the neutral CP-even, and the neutral CP-odd fields, respectively. The fields with the same quantum numbers can mix, and the resulting mass eigenstates correspond to two CP-even Higgs bosons, $h$ and $H$, where $h$ denotes the lighter CP-even Higgs boson, one CP-odd Higgs boson $A^{0}$, two charged Higgs bosons $H^{ \pm}$, and a neutral and two charged Goldstone bosons, $G^{0}$ and $G^{ \pm}$.

The original set of parameters of the Higgs and gauge sector,

$$
m_{11}^{2}, \quad m_{22}^{2}, \quad m_{12}^{2}, \quad \lambda_{1}, \quad \lambda_{2}, \quad \lambda_{4}, \quad g_{1}, \quad g_{2}, \quad \lambda_{3}, \quad \lambda_{5}, \quad\left(v_{1}, \quad v_{2},\right)
$$

with $g_{1}$ and $g_{2}$ being the $U(1)$ and the $S U(2)$ gauge coupling, respectively, is replaced by

$$
M_{h}, \quad M_{H}, \quad M_{A}, \quad M_{H^{+}}, \quad M_{W}, \quad M_{Z}, \quad e, \quad \tan \beta, \quad \alpha\left(\text { or } \lambda_{3}\right), \quad \lambda_{5}, \quad\left(t_{h}, t_{H}\right)
$$


with $t_{h}$ and $t_{H}$ being the tadpole parameters ${ }^{1}$. The masses of the CP-even, CP-odd, and charged Higgs bosons are $M_{h}, M_{H}, M_{A}, M_{H^{+}}$, the masses of $Z$ and the $W$ bosons are $M_{W}$ and $M_{Z}$. The electric unit charge is denoted by $e$. The parameter $\tan \beta$ is defined as the ratio of the two Higgs vacuum expectation values, $\tan \beta=\frac{v_{2}}{v_{1}}$. In the applied renormalization schemes ${ }^{2}$, either the quartic coupling $\lambda_{3}$ or the mixing angle $\alpha$ of the CP-even Higgs bosons is used as an input.

In all renormalization schemes,

- the masses of the Higgs bosons as well as of the electroweak gauge bosons have been chosen on-shell,

- the electric charge is fixed via the electron-positron-photon vertex ee $\gamma$ in the Thomson limit,

- the quartic coupling $\lambda_{5}$ is treated as $\overline{\mathrm{MS}}$ parameter.

Treatment of tadpoles:

$\overline{\text { In the renormalization }}$ procedure, the bare tadpole parameter $t_{\phi}^{\text {bare }}$ with $\phi=h, H$ is replaced by the renormalized tadpole parameter $t_{\phi}^{\text {ren }}$ and the corresponding counterterm $\delta t_{\phi}, t_{\phi}^{\text {bare }}=t_{\phi}^{\text {ren }}+\delta t_{\phi}$. In the applied renormalization schemes, we used two different treatments of the tadpoles:

1. "Parameter-Renormalized Tadpole Scheme", (PRTS):

The renormalized tadpole parameters are chosen to vanish, $t_{\phi}^{\text {ren }}=0$, so that the corresponding counterterm $\delta t_{\phi}$ cancels the generic one-loop tadpole contributions. Therefore, no explicit tadpole contributions have to be taken into account throughout the calculation. However, this treatment introduces gauge dependences in the relation between bare parameters [8,9], and, thus, in the relation between renormalized parameters and physical predictions.

2. "Fleischer-Jegerlehner Tadpole Scheme", (FJTS):

The bare tadpole parameters are fixed to be zero $[12]^{3}, t_{\phi}^{\text {bare }}=0$. Applying this choice, gauge dependences in the relation between bare parameters are avoided, and, hence, the relation between the renormalized parameters and physical predictions will be gauge independent. This treatment requires that explicit tadpole contributions are taken into account. However, the same set-up including tadpole counterterms as in the PRTS can be used as long as finite contributions occurring due to the different treatment of the tadpoles in the $\overline{\mathrm{MS}}$ counterterms of $\alpha$ or $\lambda_{3}$, and $\beta$ are taken into account, see Ref. [14] for a detailed description of the procedure. Via a shift of the Higgs fields, which can be interpreted as an unobservable change of the integration variables in the path integral, also in this prescription, $t_{\phi}^{\text {ren }}=0$ can be reinforced $[8,9,10,11,12,15]$.

Several different treatments of $\beta$ and either $\alpha$ or $\lambda_{3}$ have been applied. More details about the following four renomalization schemes can be found in Ref. [14].

1. $\overline{\mathrm{MS}}\left(\lambda_{3}\right)$ scheme:

In this scheme, $\lambda_{3}$ and $\beta$ are chosen as independent parameters and fixed in the $\overline{\mathrm{MS}}$ scheme,

\footnotetext{
${ }^{1}$ The parameters in brackets are not independent.

${ }^{2}$ Further renormalization schemes of the THDM are discussed in Refs. [4, 5, 6, 7, 8, 9, 10, 11].

${ }^{3}$ In Ref. [13], a similar scheme, called $\beta_{h}$ scheme, was suggested.
} 


\begin{tabular}{|c|c|c|c|c|c|c|c|}
\hline & $\alpha$ & $\lambda_{3}$ & $\beta$ & PRTS & FJTS & OS conditions wrt: & $\begin{array}{c}\text { for details, } \\
\text { see }\end{array}$ \\
\hline$\overline{\mathrm{MS}}\left(\lambda_{3}\right)$ scheme & & $\overline{\mathrm{MS}}$ & $\overline{\mathrm{MS}}$ & $\mathrm{x}$ & & & [14] \\
\hline$\overline{\mathrm{MS}}(\alpha)$ scheme & $\overline{\mathrm{MS}}$ & & $\overline{\mathrm{MS}}$ & $\mathrm{x}$ & & & [14] \\
\hline $\mathrm{FJ}(\alpha)$ scheme & $\overline{\mathrm{MS}}$ & & $\overline{\mathrm{MS}}$ & & $\mathrm{x}$ & & [14] \\
\hline $\mathrm{FJ}\left(\lambda_{3}\right)$ scheme & & $\overline{\mathrm{MS}}$ & $\overline{\mathrm{MS}}$ & & $\mathrm{x}$ & & [14] \\
\hline OS1 scheme & OS & & OS & & & $\frac{\mathscr{M}_{\left\{H, A^{0}\right\} \rightarrow v_{1} \bar{v}_{1}}}{\mathscr{M}_{h \rightarrow v_{1}} \bar{v}_{1}}$ & [15] \\
\hline OS2 scheme & OS & & OS & & & $\frac{\mathscr{M}_{\left\{H, A^{0}\right\} \rightarrow V_{2} \bar{v}_{2}}}{\mathscr{M}_{h \rightarrow V_{2}} \bar{v}_{2}}$ & [15] \\
\hline OS12 scheme & OS & & OS & & & $\begin{array}{l}\frac{\mathscr{M}_{H \rightarrow v_{2} \bar{v}_{2}}}{\mathscr{M}_{h \rightarrow v_{2} \bar{v}_{2}} \text { form factors }} \\
\text { for } h, H, A^{0} \rightarrow v_{1} \bar{v}_{1}, v_{2} \bar{v}_{2}\end{array}$ & [15] \\
\hline BFM scheme & BFM & & BFM & & $\mathrm{x}$ & $\Sigma_{h H}, T_{h}, T_{H}$ & [15] \\
\hline
\end{tabular}

Table 1: Overview about the differences in the different renormalization schemes. It should be noted that the $\overline{\mathrm{MS}}$ counterterms depend on the choice of the tadpole scheme, i.e. whether the renormalized tadpole parameters $t_{\phi}^{\text {ren }}$ vanishes by a pure parameter renormalization in the PRTS or the bare tadpole parameter $t_{\phi}^{\text {bare }}$ are chosen to vanish $(\phi=h, H)$ in the FJTS.

and PRTS for the tadpoles. The mixing angle $\alpha$ can be calculated from $\lambda_{3}$ and the other independent parameters using tree-level relations. In this scheme, within the class of $R_{\xi}$ gauges at NLO, the relations between independent parameters and predicted observables do not depend on the gauge parameter, since $\lambda_{3}$ is a basic coupling in the Higgs potential and thus does not introduce gauge dependences, and since the $\overline{\mathrm{MS}}$ renormalization of $\beta$ is gaugeparameter independent in $R_{\xi}$ gauges at NLO [8,9]. It should, however, be noted that this scheme leads to an unphysical singularity for $\cos (2 \alpha)=0$.

2. $\overline{\operatorname{MS}}(\alpha)$ scheme:

The only difference to the $\overline{\operatorname{MS}}\left(\lambda_{3}\right)$ scheme is that now $\alpha$ is chosen as independent parameter instead of $\lambda_{3}$. This scheme suffers from some gauge dependence in the relation between renormalized parameters and predicted observables. Therefore, for a meaningful comparison with data, all predictions using this renormalization scheme should be performed in the same gauge. We have applied the 't Hooft-Feynman gauge.

3. $\mathrm{FJ}(\alpha)$ scheme:

In this scheme as in the $\overline{\mathrm{MS}}(\alpha)$ scheme, $\alpha$ and $\beta$ are independent parameters, but the tadpoles are treated following the gauge-independent FJ prescription, FJTS. Similar schemes are also described in Refs. [8, 9], however, the treatment of $m_{12}^{2}$ and $\lambda_{5}$ differs from ours. 


\section{4. $\mathrm{FJ}\left(\lambda_{3}\right)$ scheme:}

In this scheme $\beta$ and $\lambda_{3}$ are independent parameters, as in the $\overline{\mathrm{MS}}\left(\lambda_{3}\right)$ scheme, but the FJTS for the tadpole parameters is chosen.

The parameters $\alpha, \beta$, and the Higgs-quartic-coupling parameter $\lambda_{5}$ depend on a renormalization scale $\mu_{\mathrm{r}}$ in the four schemes described above. The $\mu_{\mathrm{r}}$ dependence of $\alpha, \beta$, and $\lambda_{5}$ was calculated by solving the renormalization group equations in the four different renormalization schemes [14].

In Ref. [15], further renormalization schemes have been proposed, which only differ in the treatment of $\alpha$ and $\beta$ from the ones presented before. In order to avoid gauge dependences as well as a large renormalization-scale dependence of the parameters, on-shell renormalization conditions have been suggested exploiting ratios of matrix elements $\mathscr{M}$ that are defined on-shell. These matrix elements should be evaluated in the physical phase-space region, which is for example problematic for the ratio $\mathscr{M}_{H \rightarrow Z Z} / \mathscr{M}_{h \rightarrow Z Z}$, if $h$ is SM like. In Ref. [15], this problem is circumvented by introducing new fields and decoupling them. For the case of the THDM, two right-handed neutral fermion singlets $v_{1_{R}}$ and $v_{2_{R}}$ are added, and an additional $Z_{2}$ symmetry with $v_{1_{R}} \rightarrow-v_{1_{R}}$ and $v_{2_{R}} \rightarrow v_{2_{R}}$ is imposed such that $v_{1_{R}}$ couples only to the Higgs doublet $\Phi_{1}$ and $v_{2_{R}}$ only to $\Phi_{2}$. The corresponding Lagrangian is then

$$
\mathscr{L}_{v_{R}}=\mathrm{i} \bar{v}_{\mathrm{jR}} \partial v_{\mathrm{jR}}-\left[\mathrm{y}_{v_{\mathrm{j}}} \overline{\mathrm{L}}_{\mathrm{jL}}\left(\mathrm{i} \sigma_{2} \Phi_{\mathrm{j}}^{*}\right) v_{\mathrm{jR}}+\text { h.c. }\right]
$$

with an implicit sum over $j=1,2$ and $L_{j L}=\left(v_{j}, \ell_{j}\right)^{T}$ being the left-handed lepton doublet of SM and $\mathrm{y}_{v_{j}} \rightarrow 0$ in the exploited decoupling limit. The following variants of these on-shell renormalization schemes have been applied:

1. OS1 scheme:

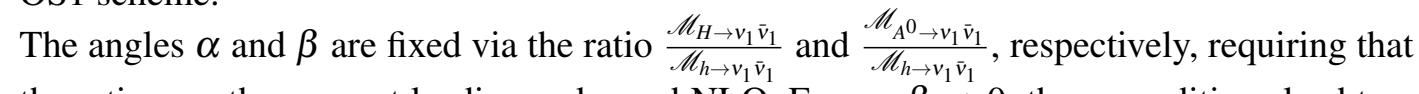
the ratios are the same at leading-order and NLO. For $\cos \beta \rightarrow 0$, these conditions lead to a singularity for $\cos \beta \rightarrow 0$ for the parameter $\beta$.

2. OS2 scheme:

For this scheme, the renormalization conditions are the same as of the above scheme but $v_{1}$ replaced by $v_{2}$. The resulting renormalization condition for $\beta$ is $\operatorname{singular}$ for $\sin \beta \rightarrow 0$.

3. OS12 scheme:

In this renormalization scheme, $\alpha$ is defined via the ratio $\frac{\mathscr{M}_{H \rightarrow v_{2} \bar{v}_{2}}}{\mathscr{M}_{h \rightarrow v_{2} \bar{v}_{2}}}$ similar to the second on-shell scheme while for $\beta$ the expression $\frac{F^{A^{0} \rightarrow v_{1} \bar{v}_{1}}}{c_{\alpha} F^{H \rightarrow v_{1} \bar{v}_{1}}-s_{\alpha} F^{h \rightarrow v_{1} \bar{v}_{1}} c_{\beta}}+\frac{F^{A^{0} \rightarrow v_{2} \bar{v}_{2}}}{s_{\alpha} F^{H \rightarrow v_{1} \bar{v}_{1}}+c_{\alpha} F^{h \rightarrow v_{1}} \bar{v}_{1} s_{\beta}}$ is exploited. The form factors $F^{A^{0} \rightarrow v_{1} \bar{v}_{1}}$ and $F^{\{h, H\} \rightarrow v_{1} \bar{v}_{1}}$ are defined via the relations

$$
\begin{gathered}
\mathscr{M}_{\{h, H\} \rightarrow v_{j} \bar{v}_{j}}^{0}=\left[\bar{u}_{v} v_{v}\right]_{\{h, H\}} F^{\{h, H\} \rightarrow v_{j} \bar{v}_{j}}, \\
\mathscr{M}_{A^{0} \rightarrow v_{j} \bar{v}_{j}}^{0}=\left[\bar{u}_{v} \mathrm{i} \gamma_{5} \mathrm{v}_{v}\right]_{\mathrm{A}^{0}} \mathrm{~F}^{\mathrm{A}^{0} \rightarrow v_{\mathrm{j}} \bar{v}_{\mathrm{j}}},
\end{gathered}
$$

where $\bar{u}_{v}$ and $v_{v}$ are the spinors of the final-state fermions. The decay kinematics of the spinor chains are indicated by the indices $[\ldots]_{h / H / A^{0}}$. 
Finally, renormalization conditions exploiting the Background-Field Method (BFM) are applied as proposed in Ref. [15] where the angles $\alpha$ and $\beta$ are defined via the mixing $\Sigma_{h H}$ and the tadpoles $T_{h}$ and $T_{H}$. The renormalization condition of $\alpha$ agrees with the renormalization condition of Ref. [8] in the "on-shell pinch technique" scheme.

An overview of the differences in the four renormalization schemes is given in Tab. 1.

\section{Summary of the calculation}

In this section, we briefly describe the calculation of the decay of the light, neutral CP-even Higgs boson of the THDM into four fermions at NLO. The computer program PROPHECY4F [16, 17, 18 $]^{4}$ provides a "PROPer description of the Higgs dECaY into 4 Fermions" and calculates observables for the decay process $\mathrm{h} \rightarrow \mathrm{WW} / \mathrm{ZZ} \rightarrow 4 f$ at NLO EW+QCD in the SM. In Refs. [14, 19], we have extended PROPHECY4F by implementing the corresponding decay in the THDM using the four $\overline{\mathrm{MS}}$-like renormalization schemes above in such a way that the features of PROPHECY4F and its applicability basically remain the same. We performed two independent calculations and implementations.

- We have used a model file generated by FeynRules [20] as well as an inhouse model file.

- The amplitudes for the virtual electroweak corrections have been generated with two different versions of FeynArts [21, 22].

For the virtual QCD corrections, the THDM amplitudes have been obtained by exploiting the SM amplitudes of Refs. [16, 18] and rescaling the Higgs couplings appropriately. It should be noted that fermion masses including the bottom quark mass were treated as vanishing in general, except for the closed fermion loops where finite fermion masses were taken into account. Hence, the contribution of diagrams with a closed fermion loop coupling to the Higgs boson do not vanish. Since the fermion coupling do not only scale differently with respect to the Higgs-gauge-boson coupling but also depends on the type of the THDM, special care had to be taken in the rescaling. We have implemented four different types (Type 1, Type 2, "flipped", "lepton-specific") that differ in how the down-type and electron-type fermions couple to the two Higgs doublets. The up-type fermions, however, couple always in the same manner in all of the four types of THDM, and the largest contribution originates from the top-quark loop while the contribution from the other fermions are small. Therefore, the differences between the types are negligible.

The tree-level and the real-emission contributions were obtained by rescaling the Higgs coupling to gauge bosons by $\sin (\beta-\alpha)$ in the result of Refs. [16, 18].

- The amplitude reduction of the electroweak corrections were performed with FormCalc $[23,24]$ in one calculation and with inhouse Mathematica routines the other one.

- The $W$ and $Z$ resonances are treated in the complex-mass scheme following the prescription in Ref. [25].

\footnotetext{
${ }^{4}$ http: //prophecy4f.hepforge.org/index.html
} 

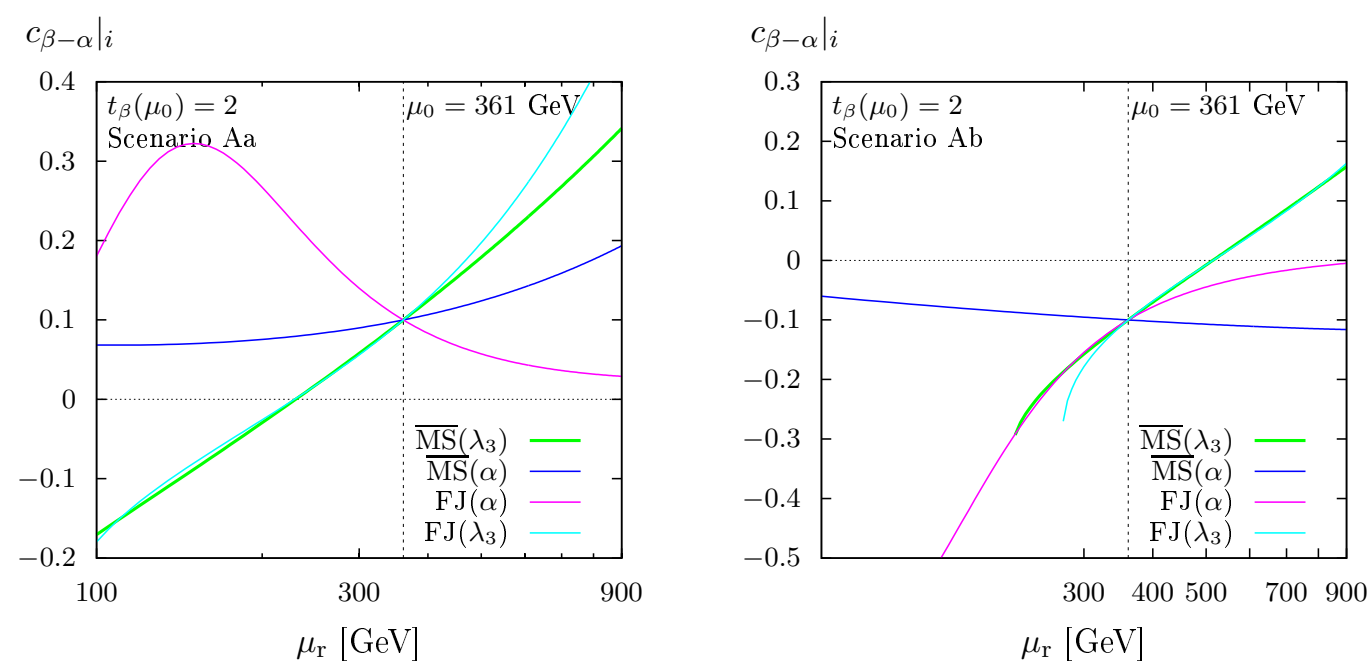

Figure 1: The renormalization scale dependence of $c_{\beta-\alpha}=\cos (\beta-\alpha)$ is shown for the four different input schemes, $\overline{\mathrm{MS}}\left(\lambda_{3}\right)$ (green), $\overline{\mathrm{MS}}(\alpha)$ (blue), $\mathrm{FJ}(\alpha)$ (magenta), and $\mathrm{FJ}\left(\lambda_{3}\right)$ (turquoise) for the scenario $\mathrm{A}$ choosing $\cos (\beta-\alpha)=0.1$ (Aa) and $\cos (\beta-\alpha)=-0.1$ (Ab) at $\mu_{0}$. It should be noted that no conversion between the different renormalization schemes has been performed for these particular plots. The plots are taken from Ref. [19].

- The evaluation of loop integrals is performed with the public Collier library [26].

- Infrared divergences have been treated applying dipole subtraction [27, 28].

More details about the calculation in the $\overline{\mathrm{MS}}$-like renormalization schemes can be found in Ref. [19]. The further extension of PROPHECY4F to the on-shell schemes, OS1, OS2, OS12, and the BFM scheme is described in Ref. [15] in detail. Moreover, Ref. [15] covers the decays $H \rightarrow W W / Z Z \rightarrow$ $4 f$ of the heavy CP-even Higgs boson.

\section{Numerical results for the partial decay width for $\mathrm{h} \rightarrow \mathrm{WW} / \mathrm{ZZ} \rightarrow 4 f$}

In this section, we show some sample results for a scenario (scenario A) inspired by Ref. [29] for the Type I THDM:

$$
M_{h}=125 \mathrm{GeV}, \quad M_{H}=300 \mathrm{GeV}, \quad M_{A_{0}}=M_{H^{+}}=460 \mathrm{GeV}, \quad \lambda_{5}=-1.9, \quad \tan \beta=2 .
$$

Within our calculation, we choose the central renormalization scale as the average mass of all scalar degrees of freedom, $\mu_{0}=\left(M_{h}+M_{H}+M_{A_{0}}+2 M_{H^{+}}\right) / 5$.

In Fig. 1, the renormalization scale dependence of the parameter $\cos (\alpha-\beta)$ is shown for the four $\overline{\mathrm{MS}}$-like renormalization schemes. In the left plot, $\cos (\beta-\alpha)=0.1$ defining the scenario Aa and in the right plot, $\cos (\beta-\alpha)=-0.1$, which is the scenario $\mathrm{Ab}$. One can clearly see that the running effects are sizeable and have different qualitative behaviour in the different schemes. It should, however, be noted that, in these plots of Fig. 1, no conversion of the parameters have been performed and that, hence, all values have to be interpreted in the respective scheme. 


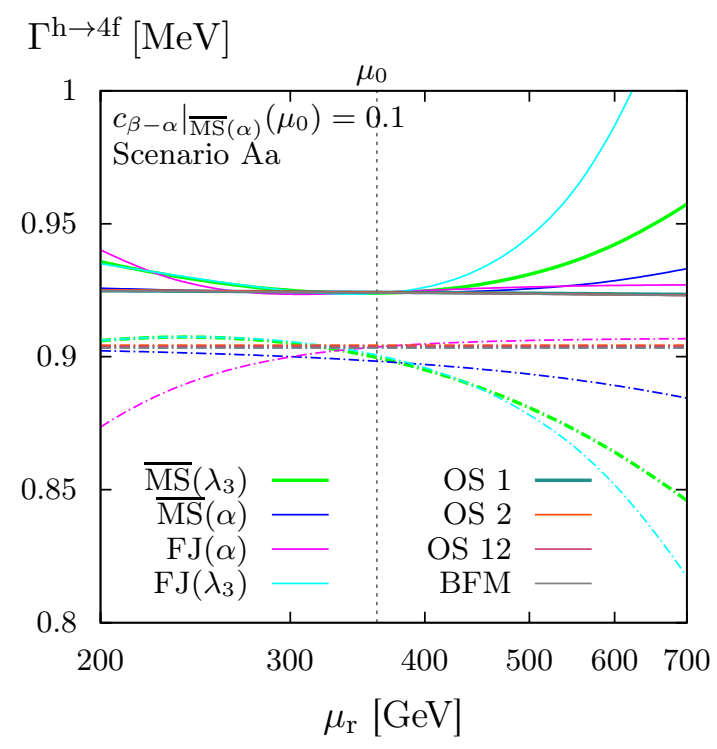

Figure 2: The renormalization scale dependence of the partial decay width $\Gamma_{\mathrm{THDM}}^{\mathrm{h} \rightarrow 4 f}$ for the scenario A given in the $\overline{\mathrm{MS}}(\alpha)$ scheme. The parameters are converted in all other schemes, $\overline{\operatorname{MS}}\left(\lambda_{3}\right)$ (green), $\overline{\operatorname{MS}}(\alpha)$ (blue), $\mathrm{FJ}(\alpha)$ (magenta), FJ $\left(\lambda_{3}\right)$ (turquoise), OS1 (dark bluegreen), OS2 (orange), OS12 (dark red), and BFM (gray). The solid lines present the result including NLO EW corrections, and the dashed ones show the tree-level result.

In Fig. 2, the renormalization scale dependence of the partial decay width for $\mathrm{h} \rightarrow \mathrm{WW} / \mathrm{ZZ} \rightarrow$ $4 f, \Gamma_{\mathrm{THDM}}^{\mathrm{h} \rightarrow 4 f}$, which is obtained by summing over all partial widths of the $\mathrm{h}$ boson with massless four-fermion final states, is shown. We fix $\cos (\beta-\alpha)=c_{\beta-\alpha}=0.1$ (scenario Aa). The parameters are given in the $\overline{\mathrm{MS}}(\alpha)$ scheme and converted to the other schemes accordingly. The dashed curves represent the LO results, however, it should be noted that the input parameters have been converted to the respective scheme denoted by the different line colours. Hence, the strict LO result is only represented by the blue $\overline{\mathrm{MS}}(\alpha)$ line. The differences between the dashed lines at the central renormalization scale $\mu_{0}$ are only due to conversion effects, while at the other scales also the different running behaviour of the $\overline{\mathrm{MS}}$ parameters in the different schemes plays a role. It should be noted that it is important to specify not only the parameter values of a certain scenario but also the renormalization scheme in which these parameters are to be interpreted.

The solid lines show the NLO results including only the EW corrections. A clear plateau around the central renormalization scale $\mu_{0}$ is visible, and there is a clear reduction on the scale dependence going from LO to NLO.

As expected, the results within the renormalization schemes that require an on-shell definition of the angles $\beta$ and $\alpha$ show a tiny dependence on the renormalization scale due to the running parameter $\lambda_{5}$ which is always chosen $\overline{\mathrm{MS}}$ and the lines representing the OS1, the OS2, the OS12, and the BFM schemes are very close or overlap for all $\mu$.

At the scale $\mu_{0}$ at NLO, all renormalization schemes agree very well, including the schemes with on-shell defined angles $\alpha$ and $\beta$, reassuring that the choice of $\mu_{0}$ is good.

The complete NLO result including also QCD corrections is shown in Fig. 3 for the given 


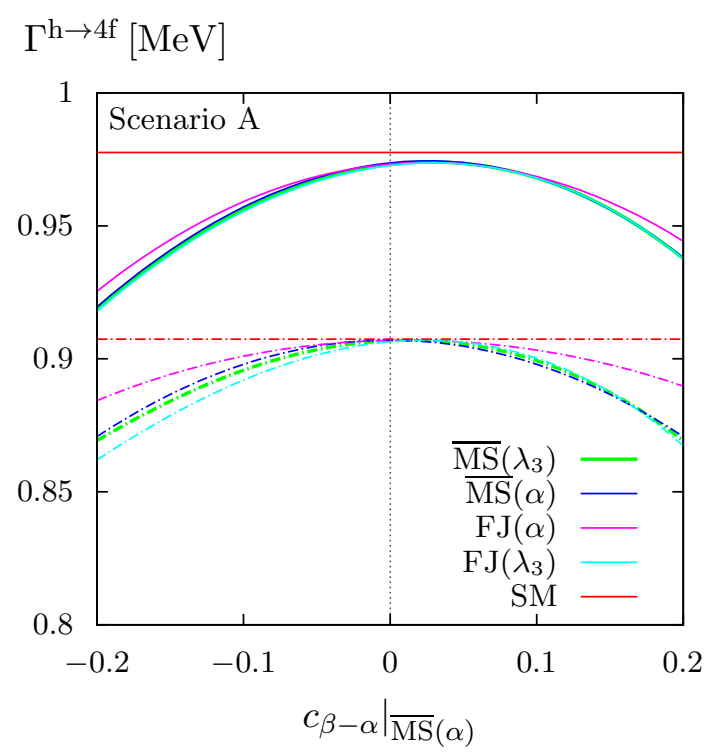

Figure 3: The dependence of the partial decay width $\Gamma_{\mathrm{THDM}}^{\mathrm{h} \rightarrow 4 f}$ on $c_{\beta-\alpha}=\cos (\beta-\alpha)$. The solid (dashed) lines represent the NLO EW + QCD (LO) result. The color code is the same as in Fig. 2. The SM results are shown in red for comparison. The input parameters are given in the $\overline{\mathrm{MS}}(\alpha)$ scheme. This figure is taken from Ref. [19].

sample scenario A exploiting the $\overline{\mathrm{MS}}$ schemes. The used input scheme is again the $\overline{\mathrm{MS}}(\alpha)$ scheme. The LO result in the $\overline{\mathrm{MS}}(\alpha)$ scheme (dashed, blue) corresponds to the SM LO results scaled by the factor $\sin ^{2}(\beta-\alpha)$ and has a parabolic shape. The deviations of the LO results in the other schemes from the $\overline{\mathrm{MS}}(\alpha)$ result are again due to the conversion of the parameters given in the input scheme to the respective target scheme. At NLO, it is interesting to note that, for all schemes, there is a deviation from the SM value also for $\cos (\beta-\alpha)=0$. This deviation originates from the heavy Higgs bosons entering the loop contributions. The overall agreement of the results in the different renormalization schemes is better at NLO than at LO.

These and further results are described in great detail in Refs. [14, 19]. For further results exploiting the OS and the BFM schemes, see Ref. [15].

\section{Conclusions}

We have calculated the partial decay widths of a Higgs boson decaying into four fermions within the THDM, $\Gamma_{\mathrm{THDM}}^{\mathrm{h} \rightarrow 4 f}$, and extended the computer program PROPHECY4F accordingly. The discussed renormalization schemes are implemented in PROPHECY4F in a way allowing for a consistent parameter conversion from one scheme to another. In addition, we took into account the running of the $\overline{\mathrm{MS}}$ parameters. The effects of the running as well of the conversion of parameters can be sizeable depending on the considered scenario. Some sample scenarios have been shown. The $\overline{\mathrm{MS}}$ results at the chosen central scale agree well with the on-shell results in the shown scenario. In scenarios where, except for the SM-like Higgs boson, the Higgs bosons are heavier than in the specifically discussed scenario, in particular, the $\overline{\mathrm{MS}}$ schemes exploiting the FJTS 
show a large scale dependence and become unreliable for certain scenarios. The schemes $\overline{\operatorname{MS}}\left(\lambda_{3}\right)$, $\mathrm{FJ}\left(\lambda_{3}\right)$, OS1, and OS2 can lead to unphysical singularities for specific parameter choices while the on-shell scheme OS12 avoids such artefacts systematically. In summary, having at hand several renormalization schemes based on different concepts ( $\overline{\mathrm{MS}}$, on-shell conditions, BFM symmetries) with proper parameter conversions between them, greatly helps to control the stability of the NLO corrections and to estimate theoretical uncertainties due to missing higher-order corrections.

The overall deviations from the prediction of the SM can be estimated by 0 to $-6 \%$ for most of the phenomenologically relevant scenarios. Hereby, NLO corrections contribute to a shift of one to two percentage points.

\section{Acknowledgement}

HR would like to thank the organizers of Corfu Summer Institute 2018 "School and Workshops on Elementary Particle Physics and Gravity" for the invitation to present this work and an interesting and enjoyable time at the Corfu Summer Institute.

\section{References}

[1] ATLAS, CMS, G. Aad et al., Phys. Rev. Lett. 114, 191803 (2015), 1503.07589.

[2] J. F. Gunion, H. E. Haber, G. L. Kane, and S. Dawson, The Higgs Hunter's Guide (Westview, 1900).

[3] J. F. Gunion and H. E. Haber, Phys. Rev. D72, 095002 (2005), hep-ph/0506227.

[4] R. Santos and A. Barroso, Phys. Rev. D56, 5366 (1997), hep-ph/9701257.

[5] S. Kanemura, Y. Okada, E. Senaha, and C. P. Yuan, Phys. Rev. D70, 115002 (2004), hep-ph/0408364.

[6] D. Lopez-Val and J. Sola, Phys. Rev. D81, 033003 (2010), 0908.2898.

[7] C. Degrande, Comput. Phys. Commun. 197, 239 (2015), 1406.3030.

[8] Krause, Marcel and Lorenz, Robin and Mühlleitner, Margarete and Santos, Rui and Ziesche, Hanna, JHEP 09, 143 (2016), 1605.04853.

[9] A. Denner, L. Jenniches, J.-N. Lang, and C. Sturm, JHEP 09, 115 (2016), 1607.07352.

[10] Krause, Marcel and Mühlleitner, Margarete and Santos, Rui and Ziesche, Hanna, Phys. Rev. D95, 075019 (2017), 1609.04185.

[11] A. Denner, J.-N. Lang, and S. Uccirati, JHEP 07, 087 (2017), 1705.06053.

[12] J. Fleischer and F. Jegerlehner, Phys. Rev. D23, 2001 (1981).

[13] S. Actis, A. Ferroglia, M. Passera, and G. Passarino, Nucl. Phys. B777, 1 (2007), hep-ph/0612122.

[14] L. Altenkamp, S. Dittmaier, and H. Rzehak, JHEP 09, 134 (2017), 1704.02645.

[15] A. Denner, S. Dittmaier, and J.-N. Lang, JHEP 11, 104 (2018), 1808.03466.

[16] A. Bredenstein, A. Denner, S. Dittmaier, and M. M. Weber, Phys. Rev. D74, 013004 (2006), hep-ph/0604011.

[17] A. Bredenstein, A. Denner, S. Dittmaier, and M. M. Weber, Nucl. Phys. Proc. Suppl. 160, 131 (2006), hep-ph/0607060. 
[18] A. Bredenstein, A. Denner, S. Dittmaier, and M. M. Weber, JHEP 02, 080 (2007), hep-ph/0611234.

[19] L. Altenkamp, S. Dittmaier, and H. Rzehak, JHEP 03, 110 (2018), 1710.07598.

[20] N. D. Christensen and C. Duhr, Comput.Phys.Commun. 180, 1614 (2009), 0806.4194.

[21] J. Küblbeck, M. Böhm, and A. Denner, Comput. Phys. Commun. 60, 165 (1990).

[22] T. Hahn, Comput. Phys. Commun. 140, 418 (2001), hep-ph/0012260.

[23] T. Hahn and M. Perez-Victoria, Comput. Phys. Commun. 118, 153 (1999), hep-ph/9807565.

[24] T. Hahn, Nucl. Phys. Proc. Suppl. 89, 231 (2000), hep-ph/0005029.

[25] A. Denner, S. Dittmaier, M. Roth, and L. H. Wieders, Nucl. Phys. B724, 247 (2005), hep-ph/0505042, [Erratum: Nucl. Phys.B854,504(2012)].

[26] A. Denner, S. Dittmaier, and L. Hofer, Comput. Phys. Commun. 212, 220 (2017), 1604.06792.

[27] S. Catani and M. H. Seymour, Nucl. Phys. B485, 291 (1997), hep-ph/9605323.

[28] S. Dittmaier, Nucl. Phys. B565, 69 (2000), hep-ph/9904440.

[29] H. E. Haber and O. Stål, Eur. Phys. J. C75, 491 (2015), 1507.04281. 